\title{
Effects of Interview Training with Simulated Patients on Suicide, Threat, and Abuse Assessment
}

\author{
By Corin L. Osborn ${ }^{*} \&$ Ralph E. Cash ${ }^{ \pm}$
}

\begin{abstract}
Mental health practitioners as often not provided sufficiently effective interview training to assess for suicidality (Schmitz et al. 2012), to evaluate for abuse (Young et al. 2001), or to respond effectively to suicidal risk (Mackelprang et al. 2014). The current study examined the efficacy of general interview training using simulated patients to increase clinician competency in assessing for threats to self or others and abuse. Data were collected from doctoral clinical psychology students, who received weekly instruction for a number of interview-relevant topics. Each participant completed pre- and post-test videotaped interviews with simulated patients. The interviews evaluated using the Skills in Psychological Interviewing: Clinical Evaluation Scales. In addition, all participants completed the Suicidal Ideation Response Inventory, Second Edition at pre-and post-test to assess their skill in responding to suicidal clients. Paired samples t-tests were used to determine differences in trainees' skills before and after the training. The tests revealed significant increases in competence for all assessment skills $(p<.001)$ as well as for response to suicidal verbalizations ( $p<.001)$. Present findings suggest broad spectrum training with simulated patients has significant implications for the efficacy of future clinicians. The implications of using simulated patients is also discussed.
\end{abstract}

Keywords: Training, Suicide, Abuse, Assessment, Competency

\section{Introduction}

As practitioners of helping professions, psychologists as well as other mental health practitioners have always had the goal of ensuring that their clients/ patients/students and others are safe, particularly from suicidal, violent, and abusive behavior. Yet mental health providers are often not provided with sufficient training to assess for suicidality (Schmitz et al. 2012), to feel confident enough to ask about or to evaluate for possible abuse (Young et al. 2001), or to be effective in responding to threats of violence and suicidal risk (Mackelprang et al. 2014). This is especially concerning, considering that in 2017 in the United States (US), suicide was one of the top 10 leading causes of death across all ages, races, ethnicities, and sexes as well as the second leading cause of death for individuals between 10 and 34 years of age (Centers for Disease Control and Prevention [CDC] 2017). The World Health Organization (2019a) has

\footnotetext{
*Psychology Trainee, Nova Southeastern University, USA.

${ }^{ \pm}$Professor, North Southeastern University, USA
} 
specified that close to 800,000 people die as a result of suicide every year and that many more attempt to die by their own hands. In addition, US estimates indicate that at least one in seven children have experienced abuse or neglect in the past year (CDC 2019) and that approximately $10 \%$ of the elderly population in the US is abused annually (Lachs and Pillemer 2015).

Typically, psychology training programs have used peer role-playing to allow students to learn and to practice new techniques. The research has even shown that role-playing therapeutic situations can be effective at developing empathy and insight into client experiences for clinicians (Beidas et al., 2014; Meier and Davis, 2011). Unfortunately, using peers in role-play training has clear limitations. Students in the roles of clients often do not take the practice scenarios seriously, and the scenarios may not be presented realistically. Students often create therapy "issues" on the spot and frequently do not accurately portray the diagnoses or real-world situations (Kaslow et al. 2009, Meier and Davis 2011). In addition, there is evidence which suggests that peer role play is not effective, at least not for training future professionals to respond appropriately to suicidal clients (Mackelprang et al. 2014).

A few models have been proposed which focus on training for assessing suicidality specifically. These models emphasize the use of structured interviews and specific suicide-focused measures (Cramer et al., 2013). However, research suggests that most of these studies have focused on interviewing actual clients who have been diagnosed with depression and/or who have a history of previous suicide attempts or ideation, an endeavor which can be very risky for trainees, even when they are closely supervised (Cramer et al. 2013). No studies have been done regarding the efficacy of assessing for threat or abuse individually, much less all three of these topics at once.

The importance of assessing comprehensively for suicidality, threat to others, and abuse has been assumed to be understood by clinicians. Despite this assumption, or, perhaps, because of it, little research has been done to determine effective ways to train future psychologists and other mental health professionals in all of these skills without involving risk to actual clients, such as that which occurs in most practicum assignments. The current study examined the efficacy of utilizing general interview training using simulated patients to increase clinician competency in assessing for suicidality, threat to others, and abuse. The results from this study are directly applicable to doctoral clinical psychology training programs in the southeastern United States and may be further generalizable. However, further research should be done to assess and to expand the generalizability of the findings.

This paper begins by providing an introduction to the need for psychology training programs and research supporting those programs, that focus on teaching interview skills and competent assessments of suicide, threat to others, and abuse. A literature review then examines prevalence rates of suicide and abuse, psychology professional competencies, existing graduate training programs, and the use of simulated patients to enhance realism and to reduce risk. Following this, the methodology of the study, including the participants, procedures, and measures 
used, is described. Results of the study are explained as well as a discussion of the findings. Finally, the paper ends with a clear conclusions segment.

\section{Literature Review}

\section{Prevalence Rates}

Prevalence rates for suicide and abuse are concerningly high and continue to rise. In 2017 in the United States, the last year for which data are currently available, suicide was one of the top 10 leading causes of death across all ages, races, ethnicities, and sexes as well as the second leading cause of death for individuals between 10 and 34 years of age (CDC 2017). The World Health Organization (WHO 2019a) specified that close to 800,000 people worldwide die as a result of suicide every year, and that many more attempt suicide. It has been reported that suicide is the third leading cause of death for individuals between 15 and 19 years of age around the world (WHO 2019a). Training mental health professionals who are appropriately prepared to assess clients for suicidal ideation and who know how to respond appropriately to those assessments could help drastically reduce the number of deaths by suicide worldwide.

In addition, US estimates indicate that at least one in seven children have experienced abuse or neglect in the past year (CDC 2019). Globally, it is estimated that up to one billion children between two and 17 years of age experienced some form of abuse within the past year (WHO 2019b) and that one in three women have experienced physical or sexual abuse in their lifetimes (WHO, 2017). Approximately one in six individuals over 60 years old have also experienced some form of abuse in community settings within the past year (WHO 2018). Older adults are frequently hesitant to report their abuse due to fear of repercussions and retaliation, so it is believed that only one in 24 cases of elder abuse are reported (WHO 2018). For this reason, it is particularly important for mental health professionals to be trained in how to assess for abuse effectively across all age ranges.

Currently, wide-scale research does not exist examining the prevalence of threats of violence to others. This is likely due to the failure to report and to track threats as effectively as suicide and abuse are monitored. In addition, threats of violence and violent acts occur in multiple settings and forms, including domestic violence, child and elder abuse, workplace violence, terrorism, school and community shootings, honor killings, etc. It is important not to allow the lack of research result in overlooking the significance of these threats in training mental health professionals. For this reason, the current study includes evaluation of the interview training model in improving students' skills in assessing for threat to others. Further research should be done to increase awareness and to establish prevalence rates of this threat. 


\section{Training Competency}

In 2005, the American Psychological Association (APA) created the Assessment of Competency Benchmarks Work Group in order to identify appropriate levels of competency for psychology trainees at various points of their training (Fouad et al. 2009). This group established competency expectations for individuals to meet in order to demonstrate readiness for practicum, internship, and practice. Fifteen core competency domains were defined: 1) professional values and attitudes, 2) self-care, 3) scientific knowledge and methods, 4) relationships, 5) diversity awareness, 6) legal and ethical standards, 7) interdisciplinary knowledge, 8) assessment, 9) intervention, 10) consultation, 11) research, 12) supervision, 13) disseminating knowledge, 14) managementadministration, and 15) advocacy (Fouad et al., 2009). The Association of State and Provincial Psychology Boards (ASPPB) reviewed these competencies and surveyed practicing psychologists in order to create a more applied model of competency in professional psychology. This model also recognized that competency levels would differ among individuals in practicum or internship as compared to those licensed or licensed for over four years. The new framework was similar to that of the APA but less comprehensive. It included only six competency domains: 1) scientific knowledge; 2) evidence-based decisionmaking; 3) interpersonal and cultural awareness; 4) professionalism and ethics; 5) assessment; and 6) intervention, supervision, and consultation (Rodolfa et al. 2013).

The purpose of competency models is to ensure that practicing psychologists are providing appropriate services to the community and to hold them accountable for doing so. A key component of the benchmarks requires that students be monitored by faculty and supervisors in order to ensure that the students are meeting the requirements. The faculty members and supervisors are expected to have ongoing conversations with students regarding their progress toward attaining these competencies and to intervene or to provide additional resources to students who are struggling (Fouad et al. 2009). This implies that universities and training programs should have reliable and valid ways to measure the competencies, to set minimal levels of attainment (MLAs) of each competency, to track student progress toward attaining the MLAs.

\section{Graduate Training Models}

Few procedures exist for outlining clear training models for psychology student clinical practices. Even fewer have been proposed offering a standard of training for interview skills, not to mention to assess for suicide, threat to others, or abuse. The limited plans that do exist rely heavily on the use of structured interviews or specific suicide-focused measures (Cramer et al. 2013). The difficulty with depending on structured interviews in training is that it does not teach the students how to ask questions on their own; to probe for more information; to respond to negative reactions from clients; or, most importantly, to use clinical judgement appropriately in situations in which suicidality, threat to 
others, and/or abuse are present. As a result, these students may go on to become licensed providers who do not feel they have sufficient training to assess for suicidality (Schmitz et al. 2012) or possible abuse (Young et al., 2001), and who, therefore lack the confidence to do so. This in turn leads to inability to respond effectively to expressions of suicidality (Mackelprang et al. 2014), threat to others, and abuse accounts.

The ability to assess clients effectively through initial interviews has repeatedly been considered an essential competency for psychologists; yet, it is infrequently addressed in training models. Interviews are a key part of how clinicians learn about their clients and begin their assessments but may be overlooked or inadequately addressed in the training process. Recent research regarding how to train individuals in tactfully and effectively conducting threat assessment interviews exists for police officers but not for psychologists (Dando and Bull 2011, Dando et al. 2008). Interested readers should refer to the work of Coral J. Dando for more information surrounding threat assessment interview training for police officers.

Psychology training programs have long used role-play experiences with peers in order to aid student development, particularly in reference to utilizing micro-counseling skills. These role-plays allow student peers to take on the role of the client or therapist in order to practice and to learn new skills. Research suggests that role-playing provides the opportunity for the student clinician to develop empathy and to gain insight into the experience of the client (Meier and Davis 2011). Unfortunately, when student peers play the parts of clients, this often results in variable and inaccurate portrayals of diagnoses, resulting in diminished effectiveness (Kaslow et al. 2009, Meier and Davis 2011). Furthermore, the practice sessions are often not taken seriously by students, and interaction with one's peers often reduces or precludes objectivity during the role-play (Kaslow et al. 2009).

\section{Use of Simulated Patients}

While role-play with peers has clear limitations, research suggests that using simulated patients (SPs) helps to eliminate these problems. SPs are actors and actresses who are trained to portray a set of symptoms consistently across various clinical interactions (Barrows 1993). This training allows the SPs to provide training experiences that are more representative of actual client interactions than role-playing with peers' permits. Medical schools have been using SPs to train their students since 1963 (Barrows 1993) and other professions, including nursing (Cangelosi 2008), nutrition (Beshgetoor and Wade 2007), pharmacy (Mesquita et al. 2010), and occupational therapy (Giles et al. 2014), have begun to do the same. In addition to its use in all of these fields, practice with SPs has been viewed as a potentially useful tool for training early in mental health clinicians' clinical training programs, assisting in the clinicians' competency acquisition as well as the evaluation of those competencies (Kaslow et al. 2009, Masters et al. 2015, Yap et al. 2012). At least $80 \%$ of medical schools in the United States are currently using SPs for both training and assessment of their students' competencies (Clay et 
al. 2000). Further research has been needed to verify the effectiveness of a training model utilizing SPs for graduate psychology programs.

The interactions clinicians have with SPs have been found to be comparable to those with real patients in genuine settings, they can be standardized, and they provide trainees with feedback from an impartial party (Kaslow et al. 2009). Having the opportunity to practice with an SP who does not actually experience the symptoms they are presenting lessens any immediate risk of harm to an actual client, reduces the probability of ethical dilemmas which might be handled poorly in a real practicum setting, and makes it more likely that the student will be adequately prepared to handle the stress of interacting with clients who are actually suffering from psychopathology. Although novice therapists report a high level of empathy for their clientele, they express considerable uncertainty over the boundaries of their newfound roles (Williams et al. 1997). Fortunately, the experience of role-playing with SPs in a safe environment increases students' confidence in their skills (Kaslow et al. 2009, Masters et al. 2015).

\section{Methodology}

\section{Participants}

Data were collected from first year doctoral clinical psychology students at a large university in the southeastern United States. One hundred doctoral psychology trainees participated in a four-month (one semester) interviewing course, during which they received instruction in general interviewing skills with specific modules detailing how to probe for and to respond to expressed concerns about possible suicide, violence, and abuse. In addition, they role-played 15minute diagnostic interviews with simulated patients, received feedback from their classmates and session facilitators, observed classmates doing similar role-plays, and provided feedback to classmates on their role-plays. As first year students, these individuals had little or no prior clinical experience interviewing clients. This made them ideal candidates for evaluating the effectiveness of a novel training program, without the potential for prior experience or training to influence initial assessment and development of their skill. Using this sample, therefore, improved the internal validity of the study by avoiding an extraneous variable mediating the outcomes.

Participants identified their gender as female $(n=78)$, male $(n=17)$, or not specified $(\mathrm{n}=5)$. Ages of participants ranged as follows: 20-24 $(\mathrm{n}=73), 25-29(\mathrm{n}$ $=20), 30-34(\mathrm{n}=4), 40-44(\mathrm{n}=2)$, and not specified $(\mathrm{n}=1)$. Participants reported identifying as Caucasian $(n=63)$, Hispanic/Latinx $(n=21)$, African American $(n$ $=7)$, Asian $(n=3)$, other $(n=3)$, and not specified $(n=3)$. Among these participants, 85 identified English as their first language, 11 reported Spanish as their first language, one noted Creole as the first language, and three did not specify their first language. Nine participants classified themselves as being trainees in the Ph.D. clinical psychology program and 91 participants were trainees in the Psy.D. clinical psychology program. Of both programs, 22 began their 
respective psychology training with a master's degree, 77 began with a bachelor's degree, and one participant did not specify.

\section{Procedures}

All first-year doctoral psychology trainees were enrolled in a required introductory pre-practicum interviewing course and received instruction in a broad variety of interviewing skills, including specific training in assessing for suicidality, threat, and abuse. Each participant was randomly assigned to a course section with 10 other students, and all sections met at the same time for approximately three hours each week for 10 weeks. Course facilitators were doctoral psychology interns and post-doctoral residents. These instructors rotated among sections so that each instructor taught a different group of students each week. As a part of this course, participants completed two 15-minute videotaped interviews with simulated patients. The first interview (the pre-test) took place prior to receiving any course instruction, and the second interview (the post-test) was conducted at the end of the course. Simulated patients portrayed a client with major depressive disorder (MDD) for both the pre-test and the post-test. Participants were assigned to a simulated patient at random for both the pre-test and the post-test.

Prior to each of the 10 course sessions as well as the pre-test and post-test, simulated patients were sent a detailed description of the case they were to portray. The descriptions included the client's name and age, clinical setting, initial body language and affect, presenting problems, responses to probable questions from clinicians, level of openness to be displayed, personal background information, and a timeline of relevant events (noting that the timeline might have to be adjusted based on the actual age of each SP). In addition, the simulated patients were provided with a two-hour, face-to-face training session regarding characteristics of each disorder to be portrayed and how it was to be displayed with opportunities provided for the SPs to ask questions. The training sessions were conducted by a psychology faculty member who was an expert in each of the five disorders to be simulated, i.e., one for the pre-test and post-test (MDD) as well as one disorder for each two consecutive course sessions (MDD and four others). Each disorder was portrayed for two consecutive sessions so that there would be adequate time for each of the students to complete a 15-minute practice interview with the SP followed by feedback from the facilitator and peers. In addition, the SPs completed a Simulated Patient Assessment of Clinician Effectiveness Scale (SPACES) feedback form for each student based on the SP's reactions to the interview. These forms were distributed to each student prior to the next class session. The SPACES scale was developed by the researchers in an effort to allow the SP to provide feedback on interviewer performance. The researchers modeled the inventory on the Arizona Clinical Interview Rating Scale, an instrument commonly used to evaluate the interviewing techniques of medical students (Stillman et al. 1977). Integrity (fidelity of implementation) checks were conducted for each portrayal by designated simulated patients who observed the 
others via closed-circuit television and used checklists designed by the course faculty.

The two videotaped interviews of the pre-test and the post-test were reviewed and evaluated by two randomly assigned interns and/or post-doctoral residents using the Skills in Psychological Interviewing: Clinical Evaluation Scales (SPICES). Items regarding competency in assessing suicidality, threat to harm others, and risk of abuse were pulled from the total SPICES scale for the purpose of this study. In addition, all participants completed the Suicidal Ideation Response Inventory, Second Edition (SIRI-2) prior to receiving instruction and at the end of the course to assess their skill in responding to verbal expressions of suicidal clients.

During the course, and between the videotaped sessions, participants received 30-45-minute instruction sessions once a week. A number of interview-relevant topics and skills, including assessing for suicide, threat, and abuse, were covered. Following instruction, participants engaged in 15-minute role-play interviews with simulated patients. Simulated patients rotated every two weeks and participants role-played once with each simulated patient. The cases presented by the simulated patients also changed every two weeks, and the simulated patients were trained in each case as described above. After completing the 15-minute role play, the participants then received feedback from peers and the instructor regarding their role-play. The instruction and role-play courses continued for 10 three-hour sessions over the course of the four-month semester.

\section{Measures}

\section{Skills in Psychological Interviewing: Clinical Evaluation Scales (SPICES)}

SPICES is a measure designed and devised to assess basic clinical interviewing skills. The measure consists of a total score and five subscales: ethical and legal standards and policy, professional values and attitudes, individual and cultural diversity relationships, and assessment. Developed by the researchers to evaluate clinical interviewing skills, SPICES currently consists of 26 questions which are rated on a four-point, Likert-type scale. Within each item, anchors using behavioral descriptions are provided to aid raters in accurately evaluating each participant and to reduce misunderstandings about the intent of the item. During the development of the SPICES measure, university faculty researchers generated a list of essential clinical competencies that have been identified in the literature, including such variables as the skills of reflecting, imparting genuineness, mirroring the client's expressions effectively, making appropriate eye contact, responding to nonverbal cues, and empathically normalizing the client's concerns, using the APA's Competency Benchmarks as a guide. Once the initial inventory of competencies was created, the skills were simplified into component parts and operationalized to enhance the ease with which the participants were to be evaluated.

The measure was subsequently distributed to faculty members, both in school psychology and in clinical psychology, who have been identified as expert 
interviewers based upon number of years of experience in the field, to evaluate the items. The researchers requested that the experts identify the items that they judged effectively assessed the outlined competencies, as well as those items that they viewed as redundant, unhelpful, or invalid. They were further asked to provide any other relevant feedback. Revisions were made based on their responses to increase the sensitivity of the rating scale, to eliminate items that were deemed ineffective, to increase the clarity and specificity of items, to modify factor names as recommended, and to eliminate any complex items that would not likely be observed in the target audience of beginning clinicians. The measure's utility, ease of use, and validity were subsequently evaluated by additional piloting of the measure by clinical psychologists and graduate students, within both clinical and school psychology programs. The measure was then modified based on the pilot data. Initial piloting of the data yielded internal consistency coefficients ranging from $\alpha=.84$ to $\alpha=.86$. Inter-rater reliabilities ranged from $r=.63$ to $r=.81$.

\section{Suicidal Ideation Response Inventory, Second Edition (SIRI-2)}

The SIRI-2 is a measure used to evaluate responses to suicidal clients' verbal expressions of distress and suicidal ideation, intent, and/or plans. Participants rate standardized responses to suicidal expressions in terms of appropriateness and are scored based on the degree to which their ratings match those made by expert suicidologists (Neimeyer and Bonnelle 1997). Lower scores on this scale indicate greater agreement with expert responses.

\section{Results}

Changes in competency in assessing for suicidality, threat, and abuse during pre- and post-test interviews were analyzed by comparing pre- and post-test SPICES scores on items used to rate those three competency areas. Ratings by each evaluator on the relevant items were averaged to create single pre- and posttest scores per participant. Evaluator scores at pre-test were compared and found not to be significantly different from each other ( $p>.05)$, justifying averaging these scores for analysis. Post-test scores for threat and abuse assessment were significantly different between the two evaluators $(\mathrm{p}=.001, \mathrm{p}=.021$ respectively) but not significantly different when evaluating suicide assessment ( $p>.05)$. For this reason, a third, more experienced, evaluator's ratings on the post-test were also used to calculate the averages in order to mediate these differences.

Paired samples t-tests were used to determine differences in trainees' skills in assessing for suicidality, threat of harm to others, and abuse before the beginning of classes (using the pre-test scores) and at the end of the semester's training (using post-test scores). Ninety-eight of the participants (two did not complete the post-test) received pre- and post-test scores for suicide, threat, and abuse assessment. Pre-test scores and standard deviations from SPICES four-point scales for each of these competencies varied (suicide $\mathrm{M}=2.95, \mathrm{SD}=.79$; threat $\mathrm{M}=2.18$, $\mathrm{SD}=1.13$; abuse $\mathrm{M}=1.46, \mathrm{SD}=.81$ ) as did the means and standard deviations for 
the post-test scores (suicide $\mathrm{M}=3.70, \mathrm{SD}=.35$; threat $\mathrm{M}=2.85, \mathrm{SD}=1.21$; abuse $\mathrm{M}=2.11, \mathrm{SD}=1.08$ ). Note that higher scores reflect better functioning on these means as well as on total SPICES scores. The paired samples t-test revealed significant increases in competence from pre-test to post-test for all skills $(\mathrm{p}<.001)$. The greatest improvement was found for assessing suicidality (t[97]=-8.438, $\mathrm{p}<.001)$. Significant improvement in ability to assess for threat $\mathrm{t}[\mathrm{97}]=-3.756$, $\mathrm{p}<.001)$ and abuse $(\mathrm{t}[97]=-4.792, \mathrm{p}<.001)$ also indicated clinically relevant changes in trainee competence. See Table 1 for a graphic of these scores. The aforementioned $\mathrm{t}$ scores represent an increase in participants' competency scores over time, rather than a decrease in some problematic characteristic, as typically expected in clinical studies, resulting in negative $\mathrm{t}$ scores.

A paired samples t-test was also used to evaluate differences in participants' knowledge of how to respond appropriately to expressions of suicidality. Pre- and post-test scores for 99 participants (one did not complete the final SIRI-2 measure) were compared. The average pre-test score was 53.65 with a standard deviation of 15.93, while the average post-test score was 44.91 with a standard deviation of 10.71 (See Table 1). This change in scores was found to represent a significant improvement in rating responses to expressions of suicidality $(\mathrm{t}[98]=5.314$, $\mathrm{p}<.001)$. Note that total scores indicate differences from experts' rating, so that lower scores reflect better judgment of possible responses to suicidal clients' expressions of concern and, therefore, greater competency.

Table 1. SPICES and SIRI-2 Competency Scores for Suicide, Threat, and Abuse Assessment Pre- and Post-training. Note that for SPICES Higher Scores Indicate Greater Competence on the Four-point Scale. For SIRI-2 Lower Scores Indicate Greater Competence

\begin{tabular}{|l|c|c|c|c|c|c|}
\hline & Pre-Test & Scores & Post-Test & Scores & $\mathbf{t}-$ & Test \\
\cline { 2 - 7 } & Mean & $\begin{array}{c}\text { Standard } \\
\text { Deviation }\end{array}$ & Mean & $\begin{array}{c}\text { Standard } \\
\text { Deviation }\end{array}$ & $\begin{array}{c}t \text { - } \\
\text { Score }\end{array}$ & $\begin{array}{c}p \\
\text { Value }\end{array}$ \\
\hline $\begin{array}{l}\text { SPICES Suicide } \\
\text { Assessment }\end{array}$ & 2.95 & .79 & 3.70 & .35 & $\begin{array}{c}- \\
8.438\end{array}$ & $<.001$ \\
\hline $\begin{array}{l}\text { SPICES Threat } \\
\text { Assessment }\end{array}$ & 2.18 & 1.13 & 2.85 & 1.21 & $\begin{array}{c}- \\
3.756\end{array}$ & $<.001$ \\
\hline $\begin{array}{l}\text { SPICES Abuse } \\
\text { Assessment }\end{array}$ & 1.46 & .81 & 2.11 & 1.08 & $\begin{array}{c}- \\
4.792\end{array}$ & $<.001$ \\
\hline SIRI-2 & 53.6513 & 15.93 & 44.9108 & 10.71 & 5.314 & $<.001$ \\
\hline
\end{tabular}

\section{Discussion}

The results of this study demonstrate significant improvements in student clinicians' skills in assessing for suicidality, threat, and abuse as well as in rating various responses to clients' suicidal expressions following a brief, broad interview training course. These findings support the efficacy of a training program that consists of brief instruction and repeated role-play involving simulated patients as well as feedback opportunities from peers, facilitators, and SPs. The use of simulated patients in the training program provided student clinicians with the 
opportunity to employ the skills they learned in the classroom in a realistic appearing but relatively risk-free environment.

The implications of this study include evaluating the efficacy of a novel framework for training clinical psychology students which may generalize to other mental health students and to established clinicians who want to refresh their most critical assessment and interviewing skills in a safe environment. It would be inappropriate to license clinicians who do not feel competant in assessing and responding to topics as vital and prevalent as suicide, threat, and abuse; yet, it happens every year (Mackelprang et al. 2014, Schmitz et al. 2012, Young et al. 2001). This training model provides a safe and practical way to train future clinicians in these skills and to improve their competence significantly in these critical areas. It is also important for seasoned clinicians to remain up-to-date on new methods of assessment and treatment, as evidenced, for example, by most states' requirement to obtain continuing education credits in order to maintain licensure for independent practice. This model may provide a safe way for these already credentialed clinicians to practice novel skills and/or to refresh basic interviewing competencies that are too often overlooked.

One key limitation of the present study is the lack of a control group. All participants received instruction sessions regarding assessment for suicide, threat, and abuse as well as specific training in how to respond to these issues, and all participants engaged in role-plays with simulated patients. It is possible that participants' improved competence was a result of one of these factors, alone or even other aspects of their curriculum, rather than the combination of the instruction and the role-play. Future studies should be designed to isolate these factors and to determine which is or are the active component(s) in the training or if they are additively beneficial.

In addition, future research should examine the long-term implications of this training program. Conducting a follow-up study with the current students or a unique longitudinal study would allow for a better understanding of the applicability of the training program. Such research would indicate whether improvments in assessment and response to expressions of suicidal ideation, intent, and/or plans are maintained over time and to what extent.

\section{Conclusions}

In 2017, suicide was one of the 10 leading causes of death across all ages, races, and sexes as well as the second leading cause of death for individuals between 10 and 34 years of age in the United States (CDC 2017). Yet few models have been proposed to train mental health professionals in assessing clients for suicidality. These professionals are frequently not provided with enough training to assess for suicidality adequately (Schmitz et al. 2012), much less to be confident and effective in responding effectively to expressions indicating suicidal risk (Mackelprang et al., 2014). In addition, no studies have been conducted analyzing a training program for clinicians that incorporate teaching clinicians to assess for threat or abuse. As a result, providers frequently do not feel confident enough to 
evaluate for possible abuse (Young et al. 2001) and may ignore these topics altogether. The programs that do exist regularly use students and peers as practice clients, which is not representative of true clinical experiences (Kaslow et al. 2009, Meier and Davis 2011).

The current study evaluated a novel training approach's ability to increase doctoral psychology trainees' competence in assessing for suicidality, threat, and abuse as well as their skill in responding appropriately to expressions of suicidality. This training model included one 30-45-minute instruction session for each of the variables of interest respectively as well as a number of other topics. Participants then engaged in 15-minute role-play interviews with simulated patients and feedback to peers for 10 sessions over the course of four months. The present findings suggest broad spectrum training in interviewing skills has significant implications for the efficacy of future clinicians. Despite the fact that suicidality, threat, and abuse assessments were each only discussed briefly during the course, they were practiced with simulated patients, and improvements persisted throughout.

Future research should expand on this study and assess its generalizability to licensed psychologists and other practicing mental health professionals as a way to refresh these skills, to learn new ones, and/or to gain confidence in utilizing either or both. These studies should also examine generalizability to other health care professionals such as medical doctors, who are frequently the first point of contact for individuals with suicidal or violence risk or for those who suffer from or are aware of someone who suffers from abuse. Increasing overall competence in assessing for and responding to these issues will, hopefully, lead to their reduction.

\section{References}

Barrows HS (1993) An overview of the uses of standardized patients for teaching and evaluating clinical skills. Academic Medicine 68(6): 443-451.

Beidas RS, Cross W, Dorsey S (2013) Show me, don't tell me: Behavioral rehearsal as a training and analogue fidelity tool. Cognitive and Behavioral Practice 21(1): 1-11.

Beshgetoor D, Wade D (2007) Use of actors as simulated patients in nutritional counseling. Journal of Nutrition Education and Behavior 39: 101-102.

Cangelosi PR (2008) Accelerated nursing students and theater students: Creating a safe environment by acting the part. Nursing Education Perspectives 29: 342-346.

Centers for Disease Control and Prevention (2019) Preventing Child Abuse \& Neglect Factsheet. Retrieved from www.cdc.gov/violenceprevention. [Accessed 27 April 2020].

Centers for Disease Control and Prevention (2017) 10 Leading Causes of Death by Age Group, United States-2017. Retrieved from https://bit.ly/3d0FURx. [Accessed 27 April 2020].

Clay MC, Lane H, Willis SE, et al. (2000) Using a standardized family to teach clinical skills to medical students. Teaching and Learning in Medicine 12(3):145-149.

Cramer RJ, Johnson SM, McLaughlin J, et al. (2013) Suicide risk assessment training for psychology doctoral programs: Core competencies and a framework for training. Training and Education in Professional Psychology 7(1): 1-11. 
Dando CJ, Bull R (2011) Maximizing opportunities to detect verbal deception: Training police officers to interview tactically. Journal of Investigative Psychology and Offender Profiling 8: 189-202.

Dando C, Wilcock R, Milne R (2008) The cognitive interview: Inexperienced police officers' perceptions of their witness/victim interviewing practices. Legal and Criminological Psychology 13: 59-70.

Fouad NA, Grus CL, Hatcher RL, et al. (2009) Competency benchmarks: A model for understanding and measuring competencies in professional psychology across training levels. Training and Education in Professional Psychology 3(4): S5-S26.

Giles A, Carson N, Breland H, et al. (2014) Use of simulated patients and reflective video analysis to assess occupational therapy students' preparedness for fieldwork. The American Journal of Occupational Therapy 68: S57-66.

Kaslow NJ, Grus CL, Campbell LF, et al. (2009) Competency assessment toolkit for professional psychology. Teaching and Education in Professional Psychology 3(4): S27-S45.

Lachs M, Pillemer K (2015) Elder abuse. New England Journal of Medicine 373: 19471956.

Masters K, Beacham A, Clement L, et al. (2015) Use of a Standardized Patient Protocol to Assess Clinical Competency: The University of Colorado Denver Comprehensive Clinical Competency Examination. Training and Education in Professional Psychology 9(2): 170-174.

Mackelprang JL, Karle J, Reihl KM, et al. (2014) Suicide intervention skills: Graduate training and exposure to suicide among psychology trainees. Training and Education in Professional Psychology 8(2): 136.

Meier ST, Davis SR (2011) The elements of counseling ( $7^{\text {th }}$ ed.). Belmont, CA: Brooks/ Cole.

Mesquita AR, Lyra DP Jr., Brito GC, et al. (2010) Developing communication skills in pharmacy: A systematic review of the use of simulated patient methods. Patient Education and Counseling 78: 143-148.

Neimeyer RA, Bonnelle K (1997) The suicide intervention response inventory: A revision and validation. Death Studies 21(1): 59-81.

Rodolfa E, Greenberg S, Hunsley J, et al. (2013) A competency model for the practice of psychology. Training and Education in Professional Psychology 7(2): 71-83.

Schmitz WM Jr, Allen MH, Feldman BN, et al. (2012) Preventing suicide through improved training in suicide risk assessment and care: An American Association of Suicidology Task Force report addressing serious gaps in US mental health training. Suicide and Life-Threatening Behavior 42(3):292-304.

Stillman PL, Brown DR, Redfield DL, et al. (1977) Construct validation of the Arizona Clinical Interview Rating Scale. Educational and Psychological Measurement 37(4):1031-1038.

World Health Organization (2017) Violence against Women Factsheet. Retrieved from https://bit.ly/3cWRvAY. [Accessed 27 April 2020].

World Health Organization (2018) Elder Abuse Factsheet. Retrieved from https://bit.ly/ 2GCVKWa. [Accessed 27 April 2020].

World Health Organization (2019a) Suicide Factsheet. Retrieved from https://bit.ly/33yI 5IV. [Accessed 27 April 2020].

World Health Organization (2019b) Violence against Children Factsheet. Retrieved from https://bit.ly/33waUFJ. [Accessed 27 April 2020].

Williams EN, Judge AB, Hill CE, et al. (1997) Experiences of novice trainees in prepracticum: Trainees', clients', and supervisors' perceptions of therapists' personal 
reactions and management strategies. Journal of Counseling Psychology 44(4): 390399.

Yap K, Bearman M, Thomas N, et al. (2012) Clinical psychology students' experiences of a pilot objective structured clinical examination. Australian Psychologist 47(3): 165173.

Young M, Read J, Barker-Collo S, et al. (2001) Evaluating and overcoming barriers to taking abuse histories. Professional Psychology: Research and Practice 32(4): 407414 . 